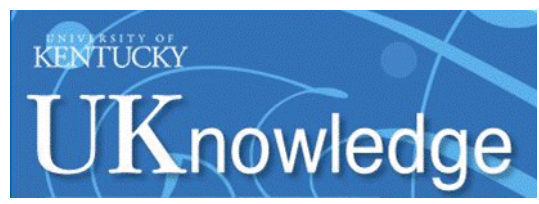

University of Kentucky

UKnowledge

9-15-1983

\title{
Stimulated Emission and the Flat Balmer Decrements of Cataclysmic Variable Stars
}

\author{
Moshe Elitzur \\ University of Kentucky, moshe@pa.uky.edu \\ Gary J. Ferland \\ University of Kentucky, gary@uky.edu \\ W. G. Mathews \\ University of California - Santa Cruz \\ G. A. Shields \\ University of Texas
}

Follow this and additional works at: https://uknowledge.uky.edu/physastron_facpub

Part of the Astrophysics and Astronomy Commons, and the Physics Commons

Right click to open a feedback form in a new tab to let us know how this document benefits you.

\section{Repository Citation}

Elitzur, Moshe; Ferland, Gary J.; Mathews, W. G.; and Shields, G. A., "Stimulated Emission and the Flat Balmer Decrements of Cataclysmic Variable Stars" (1983). Physics and Astronomy Faculty Publications. 180.

https://uknowledge.uky.edu/physastron_facpub/180

This Article is brought to you for free and open access by the Physics and Astronomy at UKnowledge. It has been accepted for inclusion in Physics and Astronomy Faculty Publications by an authorized administrator of UKnowledge. For more information, please contact UKnowledge@lsv.uky.edu. 
Stimulated Emission and the Flat Balmer Decrements of Cataclysmic Variable Stars

Digital Object Identifier (DOI)

http://dx.doi.org/10.1086/184116

Notes/Citation Information

Published in The Astrophysical Journal Letters, v. 272, no. 2, p. L55-L59.

(C) 1983. The American Astronomical Society. All rights reserved.

The copyright holder has granted permission for posting the article here.

This article is available at UKnowledge: https://uknowledge.uky.edu/physastron_facpub/180 
The Astrophysical Journal, 272:L55-L59, 1983 September 15

(1) 1983. The American Astronomical Society. All rights reserved. Printed in U.S.A.

\title{
STIMULATED EMISSION AND THE FLAT BALMER DECREMENTS OF CATACLYSMIC VARIABLE STARS
}

\author{
Moshe Elitzur and G. J. Ferland \\ Department of Physics and Astronomy, University of Kentucky, Lexington \\ W. G. MatheWS \\ Lick Observatory, University of California, Santa Cruz \\ AND \\ G. A. SHIELDS ${ }^{1}$ \\ Department of Astronomy, University of Texas, Austin \\ Received 1982 July 26; accepted 1983 May 11
}

\begin{abstract}
Balmer emission lines from cataclysmic variables often have nearly equal intensities rather than the rapid decrement predicted by simple nebular theory. Traditionally, this has been interpreted in terms of local thermodynamic equilibrium emission from a dense gas with small volume located just above the accretion disk. In this Letter we show that the intense radiation field within a close binary system can affect excited-state populations and optical emission in ways which allow a relatively low density gas to closely mimic the high-density situation. In at least one case, the old nova V603 Aql, the emitting gas has a low density and nearly fills the orbital plane of the system. If this is characteristic of other systems, then the determination of orbital parameters and masses of cataclysmic variables from emission-line radial velocities, as well as the prediction of soft X-ray emission from accreting binaries, will be affected.
\end{abstract}

Subject headings: radiation mechanisms - stars: emission-line - stars: variables

\section{INTRODUCTION}

Several classes of astronomical objects have Balmer emission lines with "flat" decrements, $I(\mathrm{H} n) / I(\mathrm{H} \beta)$ 1. Examples include old novae (Williams 1980), dwarf novae (cf. Kiplinger 1979), AM Her (Stockman et al. 1977), novae in the early phases of the outburst (e.g., Tomkin, Woodman, and Lambert 1976), and Be stars (Burbidge and Burbidge 1953). These Balmer decrements can be understood in terms of local thermodynamic equilibrium (LTE) emission at high densities (e.g., Williams 1980; Stockman et al. 1977). However, in at least one case, the old nova V603 Aql, spectroscopic evidence argues against the density being high enough to bring the relevant levels into LTE (Ferland et al. 1982). These authors suggest an alternative explanation based on the possibility that the radiation intensities are high enough for stimulated emission to modify the line ratios.

Although formation of hydrogen emission lines under conditions of large optical depths and high electron densities has been treated in some detail (see Capriotti 1964; Adams and Petrosian 1974; Netzer 1975, 1977; Krolik and McKee 1978; Drake and Ulrich 1980; Mathews, Blumenthal, and Grandi 1980), the case of intense radiation fields, such as exist near the accretion

'Alfred P. Sloan Foundation Fellow. disk of a cataclysmic variable, has been neglected. The issue is an important one because Balmer decrements have been used as a density indicator, and these density estimates influence predictions of both the size of the emission region and the soft X-ray flux.

In this Letter, we investigate this situation and show that strong radiation fields can indeed result in a flat Balmer decrement, and that this can occur at densities much lower than the values heretofore considered necessary. In many ways, the situation involving high photon densities mimics that with high electron densities. We present numerical results relevant to the various objects of interest and point at possible observational tests to discriminate between situations with large electron or photon densities.

\section{COMPUTATIONS}

Hydrogen line intensities were computed with the aid of a program which solves for the populations of all levels with $n \leq 10$. All collisional and radiative processes among the levels, and between them and the continuum, are included; the program then solves for the steady state populations $N_{n}$.

The model employed here is that of a uniform slab, and radiative transfer is treated using the escape proba- 
Wility formalism. Because a primary concern here is the Ifffect of stimulated emission, induced processes are "included from the beginning instead of "correcting" for them at the end. In particular, we use the full optical depths, namely

$$
\tau_{n n^{\prime}}=\frac{h \nu_{n n^{\prime}}}{4 \pi \Delta \nu} g_{n} B_{n n^{\prime}}\left(n_{n^{\prime}}-n_{n}\right) d,
$$

where the notation is standard (e.g., Elitzur 1982). A detailed description of the equations used and their derivation is presented separately (Elitzur 1983).

We have calculated a series of models with electron temperatures of $T=10,000$ and $15,000 \mathrm{~K}$, electron densities which were varied in a wide range upward of $10^{10}$ $\mathrm{cm}^{-3}$, and slab thickness between $10^{10}$ and $10^{12} \mathrm{~cm}$. These parameters bracket the physical conditions deduced for the corona of the old nova V603 Aql by Ferland et al. (1982). We use the thermal line width of $\Delta v=10 \mathrm{~km} \mathrm{~s}^{-1}$. Since it enters only in the combination $d / \Delta v$ in the definition of the optical depth ( $d$ is the slab thickness), a different line width would simply correspond to scaling of the physical dimensions.
The incident continuum is given by

$$
I_{\nu} \sim \nu^{p} \text { with } p= \begin{cases}2 & \left(\nu<10^{15} \mathrm{~Hz}\right) \\ 1.5 & \left(10^{15}<\nu<3.8 \times 10^{15} \mathrm{~Hz}\right) \\ 1 / 3 & \left(\nu>3.8 \times 10^{15} \mathrm{~Hz}\right)\end{cases}
$$

This simulates the shape expected for a thermal continuum emitted by an optically thick accretion disk (see Lynden-Bell 1969; Bath, Pringle, and Whelan 1980). The results are not sensitive to the exact form of the continuum above the ionization edge, nor to the value used for the cutoff, because of the rapid convergence of the ionization rate integrals over frequency.

The continuum flux is parameterized by the ionization parameter $U 1$ defined via

$$
U 1=4 \pi I_{0} / h c N_{e},
$$

where $I_{0}$ is the external radiation intensity at the ionization edge. For a pure power law above the ionization edge, $U 1$ is related to the ionization parameter $\Gamma$ of Krolik and McKee (1978) via

$$
U 1=\Gamma p .
$$

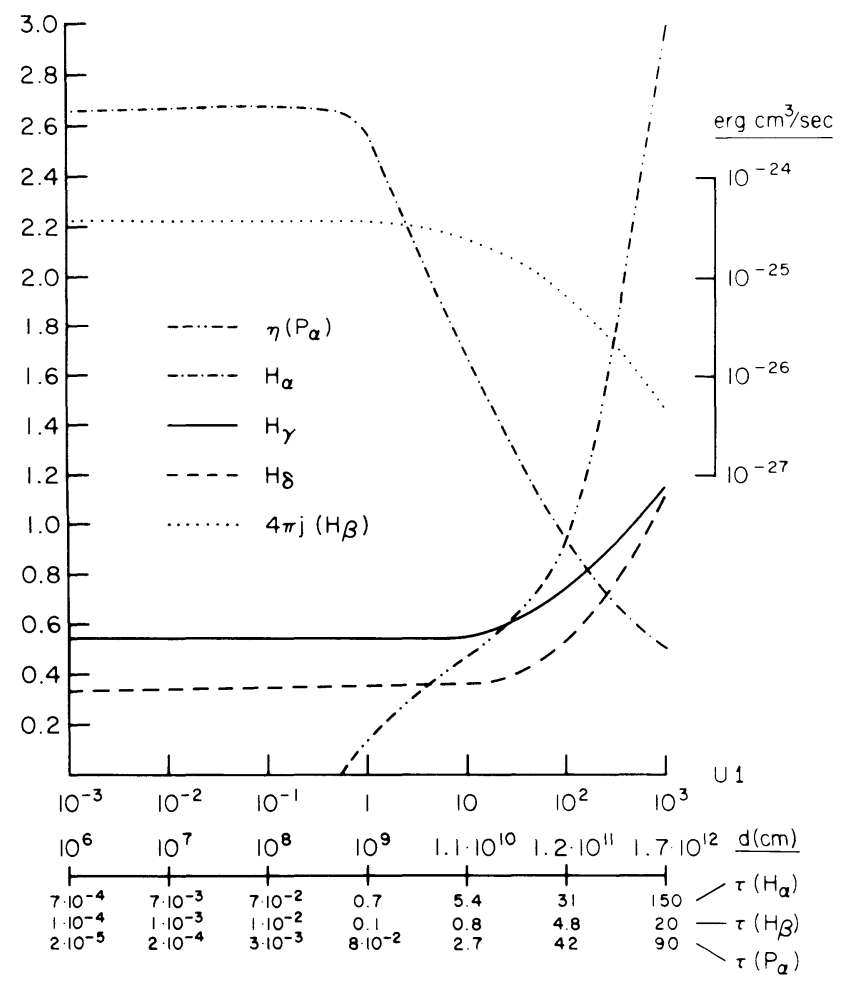

FIG. 1.-Intensities of some Balmer series emission lines, relative to $\mathrm{H} \beta$, as a function of the ionization parameter $U 1$ (see text) for a model with $T=10^{4} \mathrm{~K}, N_{e}=10^{11} \mathrm{~cm}^{-3}$, and a fixed Ly $\alpha$ optical depth of 150 . The optical depth is kept constant by varying the slab thickness $d$, which is marked on the lower axis. Also marked on the same axis are the optical depths of the $\mathrm{H} \alpha, \mathrm{H} \beta$, and $\mathrm{Pa} \alpha$ lines. The quantity $\eta(\mathrm{Pa} \alpha)$ is the photon occupation number for the internally generated radiation in the $\mathrm{Pa} \alpha$ line (see text). The scale for all the above quantities is on the left, and that for the $\mathrm{H} \beta$ emissivity $4 \pi j(\mathrm{H} \beta)$ is on the right. 
$\mathrm{Ne}=10^{11} \mathrm{~cm}^{-3} ; T=10^{4} \mathrm{~K} ; \cup 1=10^{3}$

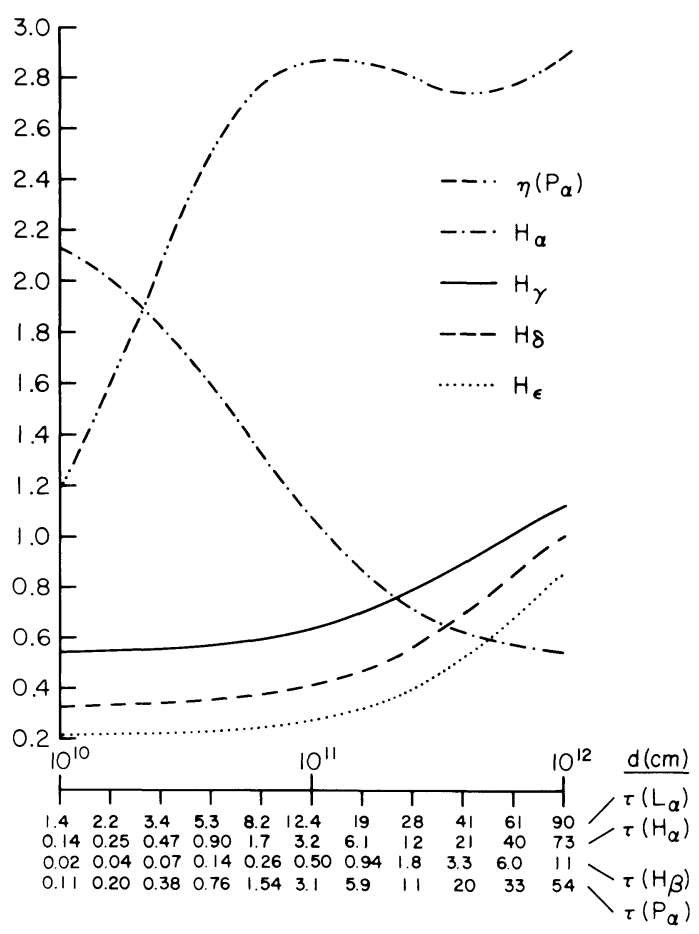

FIG. 2. - Balmer-line emissivities, relative to $\mathrm{H} \beta$, and $\mathrm{Pa} \alpha$ occupation number as a function of slab thickness $d$ for a model with $N_{e}=10^{11} \mathrm{~cm}^{-3}, T=10^{4} \mathrm{~K}$, and $U 1=10^{3}$. The lower axis shows also the corresponding $\mathrm{Ly} \alpha, \mathrm{H} \alpha, \mathrm{H} \beta$, and $\operatorname{Pa} \alpha$ optical depths.

Figure 1 shows the resulting hydrogen line ratios as a function of $U 1$ for a model with $T=10^{4} \mathrm{~K}, N_{e}=10^{11}$ $\mathrm{cm}^{-3}$, and a fixed Ly $\alpha$ optical depth of 150 . (The physical thickness of the slab is adjusted to maintain this optical depth.) At low values of $U 1$, the Balmer decrement has essentially the familiar case B values, such as $I(\mathrm{H} \alpha) / I(\mathrm{H} \beta) \approx 3$. As $U 1$ increases, the $\mathrm{H} \alpha$ intensity drops and that of the higher transitions in the Balmer series increases. The enhancement progresses to the higher lines as the intensity of the external radiation increases. Photon trapping plays a crucial role in creating this effect. As can be seen from the scale on the lower axis, the solution does not change as long as the Balmer lines are optically thin. The variation of the slab thickness, and Balmer optical depths, is due to the selection of a fixed Ly $\alpha$ optical depth since, with the other parameters held constant, the neutral fraction decreases with $U 1$. For small optical depths in the high transitions, $N_{1}$ varies inversely with, and $d$ in proportion to, $U 1$. As photon trapping in the higher lines becomes important, fewer atoms cascade to the ground state and larger increases in slab thickness are needed to maintain a fixed Ly $\alpha$ optical depth.
As evident from the figure, the "flattening out" of the Balmer decrement occurs when the Balmer lines become optically thick, and photon trapping begins to dominate the rate equations. To demonstrate the importance of induced processes in this regime, we have also plotted the occupation number for the internally generated (the "diffuse radiation") photon occupation number function for the $\mathrm{Pa} \alpha$ transition. This line is pivotal because it couples the upper levels of the $\mathrm{H} \alpha$ and $\mathrm{H} \beta$ transitions (see also Krolik and McKee 1978). It is evident that, in the region of interest, $\eta(\operatorname{Pa} \alpha)>1$ and induced processes are more important than spontaneous decays.

Also plotted is the $\mathrm{H} \beta$ emission $4 \pi j(\mathrm{H} \beta)$. The escape probability for an optically thick line is inversely proportional to the optical depth. The emissivity then becomes independent of the level populations and inversely proportional to the slab thickness, as seen in the figure.

The discussed features of the solution are also evident in Figure 2 which displays the results of a series of calculations for a model with a varying slab thickness and with $N_{e}=10^{11} \mathrm{~cm}^{-3}, T=10^{4} \mathrm{~K}$, and $U 1=10^{3}$. The decrease of $\mathrm{H} \alpha$ and increase of all the higher Balmer lines at large optical depths, as well as the importance of induced processes, are all evident.

\section{DISCUSSION}

\section{a) V603 Aquilae}

Ferland et al. (1982) analyzed optical, ultraviolet, and X-ray observations of the old nova V603 Aql. The object has a flat Balmer decrement, as is typical of cataclysmic variables (see Table 1). Previously, this had been understood in terms of emission from a small volume of dense $\left(\sim 10^{13} \mathrm{~cm}^{-3}\right)$ gas on the surface of an accretion disk $\left(R \sim 10^{11} \mathrm{~cm}\right)$. However, the International Ultraviolet Explorer observations by Ferland et al. show emission in $\mathrm{N}$ IV] $\lambda 1486$, which has a critical density $N_{\text {crit }}=10^{10.5} \mathrm{~cm}^{-3}$ for collisional deactivation. This, together with the absence of [O III] $\lambda \lambda 5007,4959$, implies that the line-emitting gas has a density $10^{9}$ $\mathrm{cm}^{-3}<N<10^{11} \mathrm{~cm}^{-3}$. They suggested a model in which the line emission comes from an extended corona with $R \sim 10^{12} \mathrm{~cm}$, comparable with the binary separation. Photoionization models gave reasonable agreement with the heavy element line intensities for a continuum from an accretion disk with $\dot{M}=10^{18} \mathrm{~g} \mathrm{~s}^{-1}$.

Table 1 shows that the calculated Balmer-line ratios for $U 1=10^{2}, N_{e}=10^{11} \mathrm{~cm}^{-3}$, and $d=10^{11.2} \mathrm{~cm}$ agree well with those observed for V603 Aql. This value of $U 1$ is derived from the observed flux and the deduced dimensions and density of the corona. The corresponding Ly $\alpha$ optical depth is 188 . Thus, within the uncertainties of the model parameters and of the observations, it is possible to explain the flat Balmer decrement as a result of the intense radiation field in the continuum and 
TABLE 1

EMISSION-LINE INTENSITIES

\begin{tabular}{|c|c|c|c|c|c|}
\hline Line & V603 $\mathrm{Aql}^{\mathrm{a}}$ & Am Her ${ }^{b}$ & SS Cyg ${ }^{c}$ & Large $J^{\mathrm{d}}$ & Large $N_{e}^{\mathrm{e}}$ \\
\hline $\mathrm{H} \alpha \ldots$ & 0.99 & 0.72 & 1.05 & 0.90 & 0.96 \\
\hline $\mathrm{H} \beta \ldots$ & 1.00 & 1.00 & 1.00 & 1.00 & 1.00 \\
\hline $\mathrm{H} \gamma \ldots \ldots$ & 0.86 & 1.15 & 0.86 & 0.81 & 0.91 \\
\hline $\mathrm{H} \delta, \ldots \ldots \ldots+\ldots, \ldots, \ldots$ & 0.92 & 0.90 & 0.77 & 0.62 & 0.82 \\
\hline $\operatorname{Pa} \alpha \ldots \ldots \ldots$ & $\ldots$ & $\ldots$ & $\ldots$ & 0.05 & 0.10 \\
\hline $\operatorname{Ly} \alpha \ldots \ldots \ldots \ldots \ldots \ldots \ldots$ & $\ldots$ & $\ldots$ & $\ldots$ & 34.5 & 14.4 \\
\hline $\operatorname{Ly} \beta \ldots \ldots \ldots \ldots \ldots \ldots$ & $\ldots$ & $\ldots$ & $\ldots$ & 11.4 & 1.99 \\
\hline $4 \pi j(\mathrm{H} \beta)\left(\operatorname{ergs~cm} \mathrm{cs}^{3} \mathrm{~s}^{-1}\right) \ldots$ & & & & $5.4 \times 10^{-26}$ & $1.34 \times 10^{-26}$ \\
\hline
\end{tabular}

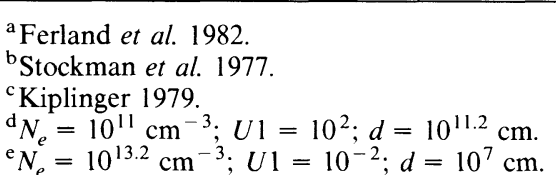

especially in the lines themselves. Densities as high as $10^{13} \mathrm{~cm}^{-3}$ are not required.

Table 1 also gives the predicted intensities of $\operatorname{Ly} \alpha$, Ly $\beta$, and $\operatorname{Pa} \alpha$. For comparison, we give a high-density model with $N_{e}=10^{13.2} \mathrm{~cm}^{-3}, U 1=10^{-2}$, and $\tau(\operatorname{Ly} \alpha)$ $=10^{4}$. Other than the external flux, which is bigger here, the parameters are those of the Drake and Ulrich (1980) model quoted by Ferland et al. (1982) as giving the best agreement with their observations of the Balmer decrement of V603 Aql. The value of $U 1$ chosen here is the smallest one that would still yield a fully ionized gas. Our calculation gives somewhat larger intensities for the higher Balmer lines than those quoted by Drake and Ulrich. This difference may be attributed to the larger ionization parameter, as well as a different treatment of the radiative transfer and of the effects of the external radiation. Although optical emission from the strong-radiation and high-density models largely mimic one another, the high-density model predicts smaller $\mathrm{Ly} \alpha / \mathrm{H} \beta$ and $\mathrm{Ly} \beta / \mathrm{H} \beta$, and larger $\mathrm{Pa} \alpha / \mathrm{H} \beta$. This suggests an observational test.

Another difference between the two models has to do with the physical dimensions of the emitting region. For the high-density model, the agreement with observations is best at a Ly $\alpha$ optical depth of about $10^{4}$, corresponding to a slab thickness of only $10^{7} \mathrm{~cm}$. (High electron densities correspond to high recombination rates which lead to large neutral densities and optical depths.) At larger dimensions, the optical depths become unacceptably high. The absence of a Balmer continuum absorption in the data presented by Ferland et al. (1982) indicates that the Balmer continuum is optically thin. This implies an $\mathrm{H} \alpha$ optical depth of less than about
$7 \times 10^{4}$. In the high-density model, such an optical depth corresponds to physical dimensions of only a few $10^{8} \mathrm{~cm}$. This contrasts with dimensions of about $10^{11} \mathrm{~cm}$ for the strong-radiation model.

\section{b) AM Herculis and SS Cygni}

Table 1 also shows an average Balmer decrement for AM Her, derived from the observations by Stockman et al. (1977), and the minimum light Balmer decrement of SS Cyg as measured by Kiplinger (1979). Both objects show a flat Balmer decrement similar to that of V603 Aql. The nature of AM Her is not well understood, and we have not attempted to explore the allowable parameters of its emission-line region. We do point out, however, that intense radiation fields may play a role in flattening the Balmer decrement of this object, and that it is not safe simply to assume an LTE emission rate for the lines. SS Cyg may be similar in many ways to V603 Aql (see the analysis by Kiplinger 1979), and it is no surprise that their emission-line spectra are similar.

We are grateful to G. H. Herbig, H. Stockman, G. R. Blumenthal, and J. Krolik for stimulating discussions. This work was supported in part by NSF grants AST 80-25222 and AST 80-23712 to the University of Kentucky, Lexington, AST $80-08246$ to the University of California, Santa Cruz, and AST 80-20461 to the University of Texas, Austin, and by NASA grant NSG 7232 to the University of Texas at Austin. G. J. F. and G. A. S. express their gratitude to Lick Observatory and the University of California, Santa Cruz, for their hospitality.

\section{REFERENCES}

Adams, W. M., and Petrosian, V. 1974, Ap. J., 192, 199

Bath, G., Pringle, J., and Whelan, J. 1980, M.N.R.A.S., 190, 185.

Burbidge, G. R., and Burbidge, E. M. 1953, Ap.J., 118, 252.
Capriotti, E. R. 1964, Ap. J., 139, 225

Drake, S., and Ulrich, R. 1980, Ap.J. Suppl. 42, 351.

Elitzur, M. 1982, Rev. Mod. Phys., 54, 1225. 
Elitzur, M. 1983, Ap. J., submitted.

Ferland, G. J., Lambert, D. L., McCall, M. J., Shields, G. A., and Slovak, M. H. 1982, Ap. J., 260, 794.

Kiplinger, A. L. 1979, Ap. J., 234, 997.

Krolik, J. H., and McKee, C. F. 1978, Ap. J. Suppl., 37, 459.

Lynden-Bell, D. 1969, Nature, 223, 690.

Mathews, W. G., Blumenthal, G. R., and Grandi, S. A. 1980, Ap. J., 235, 971.
Netzer, H. 1975, M.N.R.A.S., 171, 395. 1977, M.N.R.A.S., 178, 489

Stockman, H., Schmidt, G., Angel, R. P., Leibert, J., Tapia, S., and Beaver, E. 1977, Ap. J., 217, 815.

Tomkin, J., Woodman, J., and Lambert, D. L. 1976, Astr. Ap., 48, 319 .

Williams, R. E. 1980, Ap. J., 235, 939.

M. Euitzur and G. J. Ferland: Department of Physics and Astronomy, University of Kentucky, Lexington, KY 40506

W. G. MATHEws: Lick Observatory, University of California, Santa Cruz, CA 95064

G. A. SHIELDS: Astronomy Department, University of Texas, Austin, TX 78712 